Research Article

\title{
On the estimation of the friction factor: a review of recent approaches
}

\author{
Gabriel Plascencia $^{1}$ (D) $\cdot$ Lamberto Díaz-Damacillo $^{2} \cdot$ Minerva Robles-Agudo $^{1}$
}

Received: 21 October 2019 / Accepted: 3 January 2020 / Published online: 8 January 2020

(c) Springer Nature Switzerland AG 2020

\begin{abstract}
The friction factor is traditionally computed through Colebrook's equation or by using Moody's chart. However, these approaches have shown their limitations in getting accurate values for such factor. Different models to calculate the friction factor have been proposed, achieving different levels of certainty. In this paper, we revisit the distinct strategies used to estimate the friction factor and propose the use of a modified version of a model proposed by these authors. This model is based on the phenomenology of the flow as it transitions from laminar to turbulent flow conditions.
\end{abstract}

Keywords Friction factor · Colebrook equation · Moody chart · Lambert W function · Artificial intelligence

\section{List of symbols}

$\varepsilon \quad$ Average projection of roughness, $\mathrm{m}$

$v \quad$ Kinematic viscosity, $\mathrm{m}^{2} / \mathrm{s}$

$\varepsilon / D$ Relative roughness

$f \quad$ Friction factor

Re Reynolds number and similar abbreviations do not use italics

$D \quad$ Pipe diameter, $\mathrm{m}$

$U$ Flow maximum velocity, $\mathrm{m} / \mathrm{s}$

$\bar{u} \quad$ Flow mean velocity, $\mathrm{m} / \mathrm{s}$

$V^{*} \quad$ Shear force-velocity or friction velocity, $\mathrm{m} / \mathrm{s}$

\section{Introduction}

In determining flow rates to distribute a fluid through a network of pipes, is essential to estimate losses in kinetic energy that the flow experiences due to head loss. To do that, it is necessary to calculate the friction factor $(f)$. The friction factor relates to pressure drop and fluid viscous effects [1].

The friction factor is traditionally estimated by means of Colebrook equation or by using Moody's chart. Engineering schools worldwide teach the use of these approaches to calculate that factor; in spite of existing a whole body of literature with diverse models developed throughout time to determine that factor.

In this paper, we discuss the use of alternative models to compute the friction factor besides using Colebrook's equation and/or Moody's chart.

\section{Colebrook equation and moody's chart}

At the dawn of the last century, experiments were conducted to estimate velocity distribution in a pipe; from these tests, it was possible to determine the friction factor. Among the first results from those experiments, it was found that under the laminar regime, the friction factor decreases as Reynolds (Re) number increases:

$f=\frac{64}{\operatorname{Re}}$

Further research on the turbulent flows allowed to propose the following expression for the friction factor [2] under such regime:

$\triangle$ Gabriel Plascencia, g.plascencia@utoronto.ca; Lamberto Díaz-Damacillo, diaz.lamberto@gmail.com | ${ }^{1}$ Universidad Tecnológica de Querétaro, Av. Pie de la Cuesta 2501, Col. Nacional, 76148 Querétaro, Qro, Mexico. ${ }^{2}$ Departamento de Física, Instituto Nacional de Investigaciones Nucleares, Km 36. 5 Carretera México - Toluca, 52750 La Marquesa, Edo Mex, Mexico. 
$f=\frac{0.316}{\operatorname{Re}^{0.25}}$

Figure 1 shows plots of Eqs. (1) and (2); in the laminar region, $f$ decreases with a slope of 1 ; whereas, for the turbulent region, $f$ decreases with a slope of 0.25 .

von Kárman [3] and other researches kept looking for expressions to accurately determine the friction factor, particularly in the turbulent regime. Initially, it was proposed that the friction factor only depended on the effect of Reynolds number [4]. von Mises [5] first introduced the concept of pipe relative roughness and also described its influence on the calculation of the friction factor.

Eventually, Nikuradse [2], conducted a series of experiments to elucidate the effect of the flow's Reynolds number and the pipe wall roughness on the friction factor. To do so, Nikuradse glued sand grains of different homogeneous particle size to smooth quartz tubes. As a result, he induced a "controlled" pipe wall roughness to his experiments.

From the works of Nikuradse [2] and von Kárman [3], it was developed a general expression to determine the velocity distribution in the vicinity of the pipe wall:

$\frac{\bar{u}}{U}=\frac{\bar{u}}{U}\left(\log \left(\frac{V^{*} \varepsilon}{v}\right)\right)$

Equation (3) is a dimensionless relation that indicates that the flow velocity near the pipe walls is described by a logarithmic function.

To solve (3), it was proposed [2] a set of equations to calculate the friction factor: for rough pipes:

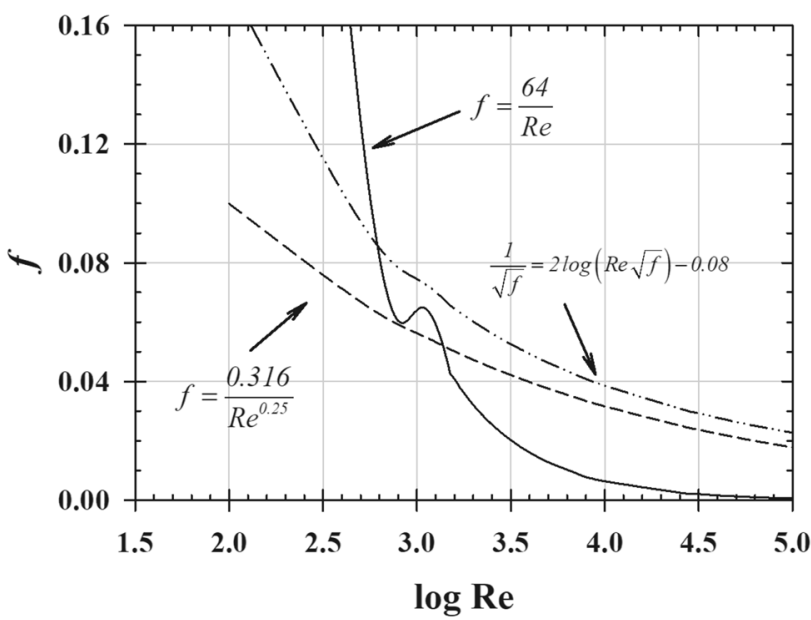

Fig. 1 Early estimations of the friction factor in the laminar and turbulent regimes

SN Applied Sciences

A SPRINGER NATURE journal $\frac{1}{\sqrt{f}}=1.14-2 \log \left(\frac{\varepsilon}{D}\right)$

Whereas for smooth pipes, it was obtained:

$\frac{1}{\sqrt{f}}=2 \log (\operatorname{Re} \sqrt{f})-0.08$

Equation (4a) only depends on the relative roughness $(\varepsilon / D)$ of the pipe wall; it does not change with increasing the value of Re; whereas Eq. (4b) only varies with Re. This equation is also plotted in Fig. 1. Equation (4b) presents a considerable deviation from Eq. (2). Such difference prompted further studies on the estimation of the friction factor.

Colebrook and White [6] conducted similar tests to those in [2] and eventually upon the integration of (3) with proper boundary conditions, Colebrook [7] came up with an expression to estimate the friction factor as a function of the relative pipe wall roughness and the flow's Reynolds number:

$\frac{1}{\sqrt{f}}=-2 \log \left(\frac{1}{3.7} \frac{\varepsilon}{D}+\frac{2.51}{\operatorname{Re}} \frac{1}{\sqrt{f}}\right)$

Equation (5) is implicit in $f$; to solve this equation, it is necessary to implement a numerical scheme. Nowadays that is simple to do due to the advent of computing; but at the time this model was proposed, the calculation of $f$ represented a serious task. To help in computing the friction factor, Moody [8] developed a graphical solution to Colebrook equation; such a solution is called Moody's chart.

Given its graphic nature, the estimation of $f$ through Moody's chart is not reliable; over or underestimations of the friction factor occur frequently. In addition to that, Colebrook and Nikuradse experiments were conducted up to Re values of $10^{6}$. Flows with higher velocities were not studied at the time, thus limiting the use of Eq. (5) to flows with Re between $10^{3}$ and $10^{6}$. Colebrook nor Nikuradse measured the friction factor under laminar flow conditions, thus Colebrook's model is inaccurate at low flow velocities.

\section{Other approaches to determine $f$}

In view of these limitations, several alternatives have been proposed over the years to estimate the friction factor. These alternatives basically consist of:

1. developing new implicit or explicit models to solve for $f$ based upon the integration of (3) or by conducting statistical analyses; 
2. the use of Lambert's W function to obtain an exact analytical solution to Colebrook's equation, and

3. the use of artificial intelligence (neural networks and symbolic regression) to compute the friction factor.

Apart from these approaches, the estimation of the friction factor has been undertaken by these authors after revisiting the physics behind the laminar to turbulent flow transition.

The strategies used to calculate the friction factor are discussed in the upcoming sections of this text.

\subsection{Models based on the integration of Eq. (3)}

Of the models reported in the literature, some of them are explicit in $f$. Explicit models tend to diverge from experimental data as recently shown [9].

On the other hand, statistical analyses of factor friction calculations with diverse models were conducted to propose a complex equation to compute $f$ [10]; the resulting model is explicit and offers relative good approximation with respect to experimental measurements.

Haaland [11] produced an explicit expression to estimate $f$; this expression contains the first term of Colebrook equation; it differs from (5) in the use of the integration limits used to integrate (3). Zigrang and Sylvester [12] also developed an equation to calculate the friction factor that also contains the term $(\varepsilon / D) / 3.7$ that is present in (5).

On the other hand, Churchill [13] proposed a so-called universal formula to estimate the friction factor over a wide range of $R e$ and relative roughness values. This model diverges from experimental values of $f$. Interestingly; Churchill's model was built differently from others. It consisted of correlating analytical solutions to fluid flow and empirical correlations. The resulting model looks complex, but the calculation of $f$ is amenable.

Recently Shaikh et al. [14] proposed another correlation to compute the friction factor. This model diverges considerably from the experimental data set [2] used for comparison. The divergence in this model is more notorious at low Re numbers and low pipe wall roughness; this may be due to the fact that the model was constructed to work under highly turbulent flow conditions and rough pipes.

Figure 2 shows the deviation of the calculated values of $f$ with the models described have with respect of the experimental data [2]; this comparison includes Colebrook's equation. The data shown in Fig. 2a considers an $\varepsilon / \mathrm{D}$ value of 0.0009861 , which corresponds to $\mathrm{r} / \mathrm{k}=507$ condition in [2]; whereas to compute the data in Fig. $2 \mathrm{~b}$ it was used a value of $\varepsilon / D=0.033333$ which corresponds to $r / k=15$ condition in [2].

These plots show the effect of pipe wall roughness on $f$; as the roughness increases, the higher is the departure of the calculated values of $f$, with respect to the measured values. Of the different models shown in Fig. 2a, b, Colebrook's offers the best fit of all of them. Figure $2 b$ shows that computed $f$ values tend to converge to the measured data as the numerical value of the friction factor increases. Such condition is attained as the flow augments its inertia, i.e. Re scales up. Low Re numbers present more deviation in the calculated friction factor with respect to the measured data.

The plots in Fig. 2a, b also indicate the effect of proper selection of integration limits to solve Eq. (3); this is the key point with Eq. (5). The manner in which Colebrook selected the integration limits allows for having such good agreement with the experimental $f$ values. Table 1 shows the expressions referred to in this section.

\subsection{Estimation of the friction factor by solving Colebrook equation analytically}

To provide better solutions for the friction factor, it has been proposed the use of Lambert's W function [15]. This function offers a mathematical tool that can be used to solve engineering problems of different nature [16], among them solving Colebrook's equation analytically rather than numerically as has been the case. W function is defined as $[16,17]$ :

$W \exp (W)=x \Leftrightarrow W=W(x)$

To solve (5) with the aid of (6), the former must be transformed is such a way that it satisfies the latter.

To transform (5) into a suitable form to apply (6), it is necessary to use dimensionless parameters. The selection of such parameters leads to possible exact solutions for Colebrook's equation. These solutions differ from author to author, obtaining different results.

For example, More [15] proposed the solution of Colebrook's equation using three parameters along with an explicit form of the Lambert $W$ function. This is shown in Table 2. More's analytical solution to Colebrook's equation is:

$$
f=\frac{1}{c\left[W_{0}\left(\frac{\exp \left(\frac{a}{b c}\right)}{b c}\right)-\frac{a}{b}\right]^{2}}
$$

To solve this expression, the $W_{0}$ term represents an explicit form of the Lambert $W$ function. This can be solved using MATLAB or MAPLE or any similar software, or it can be approximated [18] by: 
A
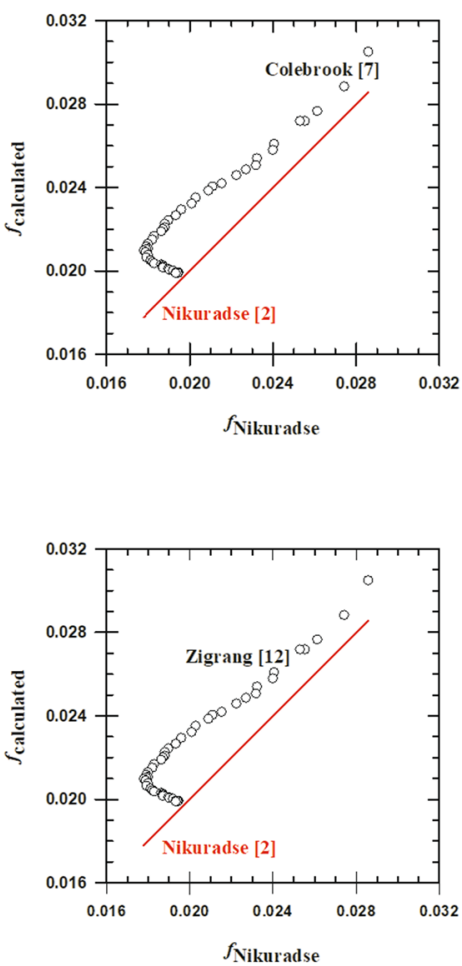

B
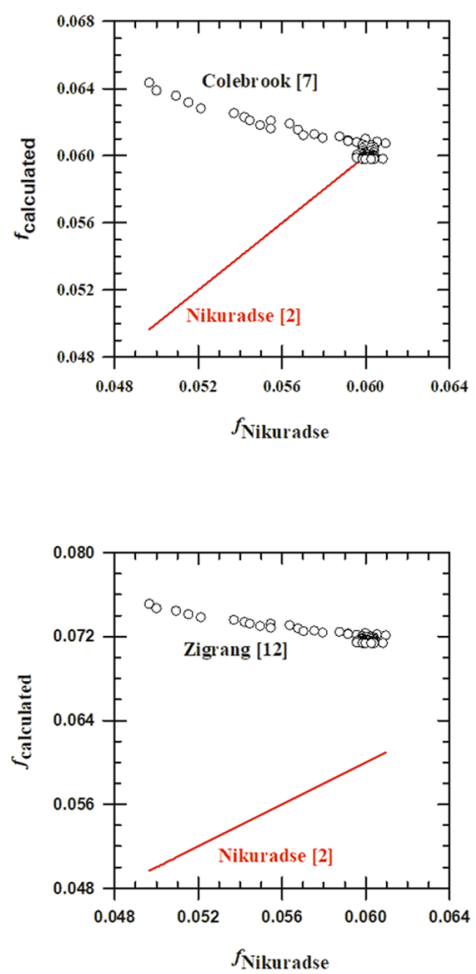

$\varepsilon / \mathrm{D}=0.000986193$
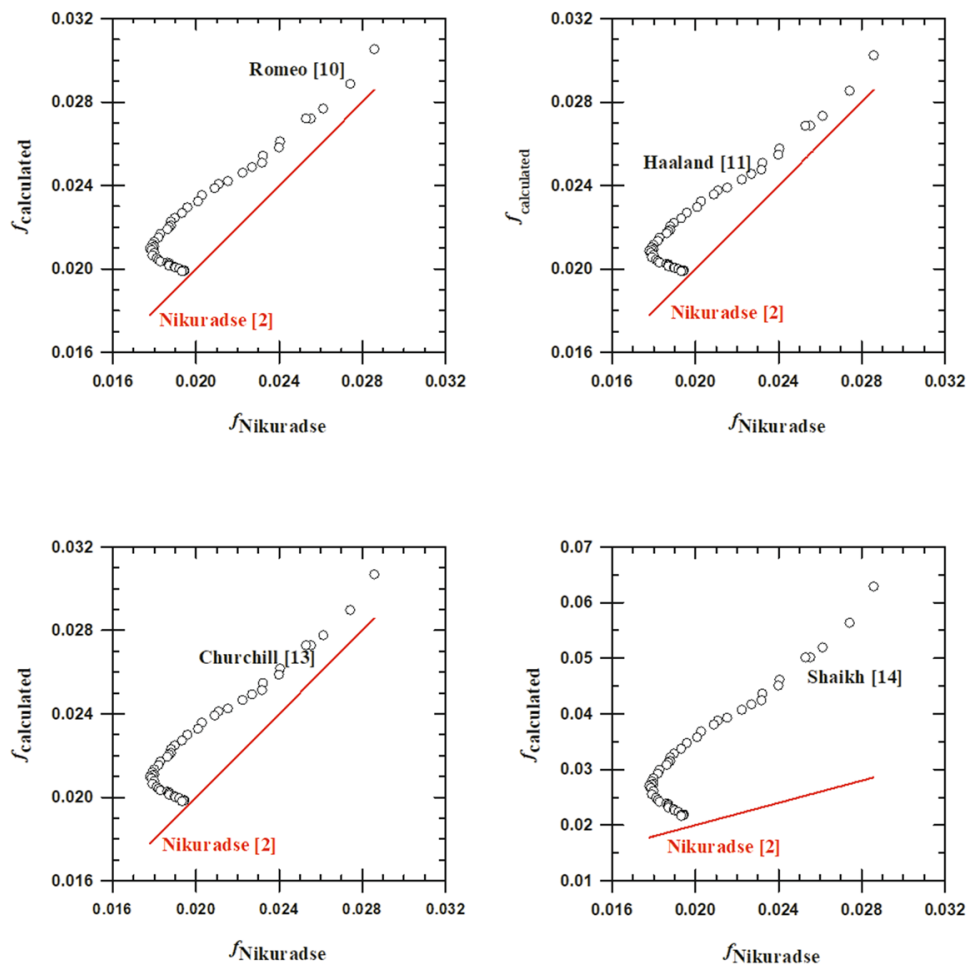

$\varepsilon / \mathrm{D}=\mathbf{0 . 0 3 3 3 3 3 3 3 4}$
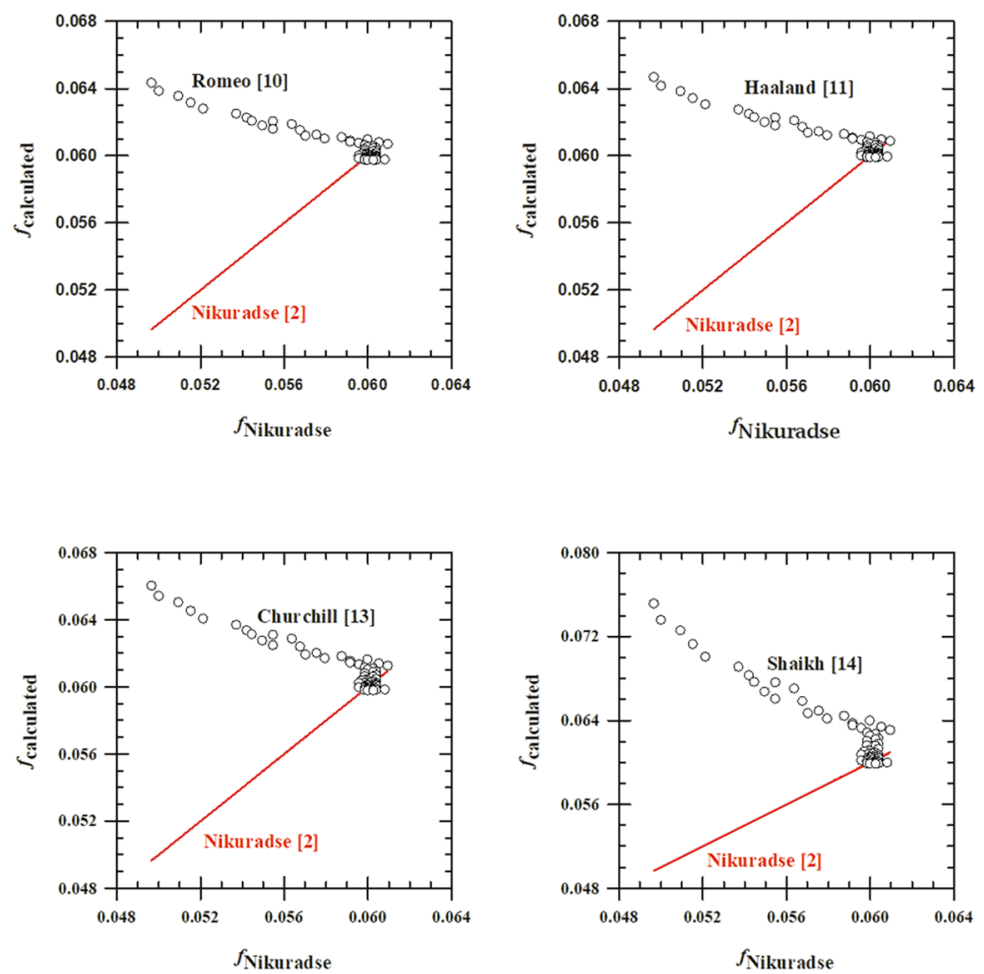

Fig. 2 Departure of calculated values of the friction factor with respect of Nikuradse data set for: $\mathbf{a} \varepsilon / D=0.00098619, \mathbf{b} \varepsilon / D=0.033333$ 
Table 1 Some available models to estimate the friction factor

\begin{tabular}{|c|c|c|c|}
\hline Ref. & Model & Validity & Error (\%) \\
\hline Colebrook [7] & $\frac{1}{\sqrt{f}}=-2 \log \left(\frac{1}{3.7} \frac{\varepsilon}{D}+\frac{2.51}{\operatorname{Re}} \frac{1}{\sqrt{f}}\right)$ & $\begin{array}{l}4 \times 10^{3}<\operatorname{Re}<10^{8} \\
0<\varepsilon / D<0.05\end{array}$ & \\
\hline Romeo [10] & $\frac{1}{\sqrt{f}}=-2 \log \left[\frac{\varepsilon}{3.7065 D}-\frac{5.0272}{\operatorname{Re}} \log \left[\frac{\varepsilon}{3.827 D}-\frac{4.567}{\operatorname{Re}} \log \left[\left(\frac{\varepsilon}{7.7918 D}\right)^{0.9924}+\left(\frac{5.3326}{208.815+\mathrm{Re}}\right)^{0.9345}\right]\right]\right]$ & $\begin{array}{l}3 \times 10^{3}<\operatorname{Re}<1.5 \times 10^{8} \\
0<\varepsilon / D<0.05\end{array}$ & 10.94 \\
\hline Haaland [11] & $\frac{1}{\sqrt{f}}=-1.8 \log \left[\frac{6.9}{\operatorname{Re}}+\left(\frac{\varepsilon}{3.7 D}\right)^{1.11}\right]$ & $\begin{array}{l}4 \times 10^{3}<\operatorname{Re}<10^{8} \\
10^{-6}<\varepsilon / D<0.05\end{array}$ & 43.86 \\
\hline Zigrang [12] & $\frac{1}{\sqrt{f}}=-2 \log \left[\frac{\varepsilon}{3.7 D}-\frac{5.02}{\operatorname{Re}} \log \left[\frac{\varepsilon}{3.7 D}-\frac{5.02}{\operatorname{Re}} \log \left[\frac{\varepsilon}{3.7 D}+\frac{13}{\operatorname{Re}}\right]\right]\right]$ & $\mathrm{N} / \mathrm{A}$ & 10.91 \\
\hline Churchill [13] & $f=8 \times\left[\left(\frac{8}{\operatorname{Re}}\right)^{12}+\frac{1}{\left[\left(2.457 \ln \left(\frac{1}{\left(\frac{7}{\text { Re }}\right)^{0.9}+0.27 \frac{\varepsilon}{D}}\right)\right)^{16}+\left(\frac{37530}{\text { Re }}\right)^{16}\right]^{\frac{3}{2}}}\right]^{\frac{1}{12}}$ & $\begin{array}{l}4 \times 10^{3}<\operatorname{Re}<10^{8} \\
10^{-6}<\varepsilon / D<0.05\end{array}$ & 11.64 \\
\hline Shaikh [14] & $f=0.25 \times\left[\log \left(\frac{2.51}{\left[1.14-2 \log \left(\frac{\varepsilon}{D}\right)\right]^{-2} \operatorname{Re}}+\frac{\varepsilon}{D} \frac{1}{3.7}\right)\right]^{-2}$ & $\begin{array}{l}10^{4}<\operatorname{Re}<10^{8} \\
10^{-4}<\varepsilon / D<0.05\end{array}$ & \\
\hline
\end{tabular}

Table 2 Expressions to calculate the friction factor using Lambert's W function

\begin{tabular}{|c|c|c|}
\hline Ref. & Solution using $\mathrm{W}$ function & Parameters used in solution with $\mathrm{W}$ function \\
\hline More [15] & $f=\frac{1}{c\left[W_{0}\left(\frac{\exp \left(\frac{a}{b c}\right)}{b c}\right)-\frac{a}{b}\right]^{2}}$ & $\begin{array}{l}a=\frac{1}{\beta \cdot 2} \frac{\varepsilon}{\operatorname{\theta }} \\
b=\frac{\text { Re }}{\text { Re }} \\
c=1.7372\end{array}$ \\
\hline Mikata [17] & $\frac{1}{\sqrt{f}}=c_{1}\left(x_{2}-\ln \left(Y\left(x_{1}\right)\right)\right)$ & $\begin{array}{l}c_{1}=\frac{2}{\ln (10)} \\
x_{2}=\ln \left[\frac{\ln (10)}{2}\left(\frac{\mathrm{Re}}{2.51}\right)\right]\end{array}$ \\
\hline & & $Y\left(x_{1}\right)=\ln \left[\frac{\ln (10)}{2} \frac{\mathrm{Re}}{2.51}\right]+\frac{\ln (10)}{2}\left(\frac{\mathrm{Re}}{2.51}\right)\left(\frac{1}{3.7} \frac{\varepsilon}{D}\right)$ \\
\hline Brkić [18] & $\frac{1}{\sqrt{f}}=-2 \log \left[\frac{2.18}{\operatorname{Re}} \beta+\frac{1}{3.7} \frac{\varepsilon}{D}\right]$ & $\beta=\ln \left[\frac{\mathrm{Re}}{1.816 \ln \left(\frac{1.1 \mathrm{Re}}{1+1.1 \mathrm{Re}}\right)}\right]$ \\
\hline Rollman [20] & $f=\left[\frac{2}{\ln (10)} W_{0}(\overline{\operatorname{Re}})-\frac{\operatorname{Re}}{(2.51)(3.71)} \frac{\varepsilon}{D}\right]^{-2}$ & $\begin{array}{l}\overline{\operatorname{Re}}=\operatorname{Re}_{1}^{*} \exp \left(\operatorname{Re}_{2}^{*}\right) \\
\operatorname{Re}_{1}^{*}=\operatorname{Re} \frac{\ln (10)}{2(2.51)} \\
\operatorname{Re}_{2}^{*}=\operatorname{Re} \frac{\ln (10)}{2(2.51)(3.71)} \frac{\varepsilon}{D}\end{array}$ \\
\hline
\end{tabular}

$W_{0}(x)=\ln \left[\frac{x}{\ln \left[\frac{x}{\ln \left[\frac{x}{\ddots}\right]}\right]}\right]$

Mikata and Walczak [17], used W function to obtain the following expression for $f$ :

$$
\frac{1}{\sqrt{f}}=c_{1}\left[x_{2}-\ln \left(Y\left(x_{1}\right)\right)\right]
$$

This expression is valid for $x_{1}>1$. The terms in (9) are depicted in Table 2.
Brkić [18] obtained an approximated solution to Colebrook's equation. The resulting expression was obtained using (8):

$\frac{1}{\sqrt{f}}=-2 \log \left[\frac{2.18}{\operatorname{Re}} \ln \left(\frac{\operatorname{Re}}{1.816 \ln \left(\frac{1.1 \operatorname{Re}}{1+1.1 \operatorname{Re}}\right)}\right)+\frac{1}{3.71} \frac{\varepsilon}{D}\right]$

Recently, a modification of Eq. (10) was proposed [19]. This results in Eq. (10a). Equation (10a) is more accurate and simpler than (10), besides, it consumes less computational resources: 


$$
\begin{aligned}
\frac{1}{\sqrt{f}} & \sim 0.8686\left[B-C+\frac{1.038 C}{0.332+B+A}\right] \\
A & =\frac{\operatorname{Re}}{8.0878} \frac{\varepsilon}{D} \\
B & =\ln (\operatorname{Re})-0.7794 \\
C & =\ln (B+A)
\end{aligned}
$$

The value of variable $a$ in (10a) is at least $10^{5}$.

Rollmann and Spindler [20], also provided an explicit solution for $f$ using Lambert's W function. Their expression for the friction factor is:

$f=\left[\frac{2}{\ln (10)} W_{0}(\overline{\operatorname{Re}})-\frac{\operatorname{Re}}{(2.51)(3.71)} \frac{\varepsilon}{D}\right]^{-2}$

Figure 3 compares the results for the friction factor obtained by using Eqs. (7), (9), (10) and (11) respectively as they are compared with Nikuradse's experimental data set and the calculated $f$ values with Colebrook equation.

From the explicit solutions shown in (7), (9), (10) and (11), it is clear that some of the terms in (5) have to be re-ordered in a suitable form to apply (6). From this set of equations, it results that the use of Lambert's W function is tricky; since if it is not used properly it leads to errors in determining accurate values for the friction factor.

In Fig. 3, for the smoothest pipe condition $(\varepsilon / D=0.0009861)$, Eqs. (9), (10) and (11) are in good agreement with Colebrook's equation and almost present the same deviation with respect of Nikuradse's experimental data. Only More's equation disagrees with respect to the other models cited.

On the other hand, when the pipe roughness increases to its maximum tested value $(\varepsilon / D=0.03333333)$, the model in [20] does not work as well as in the smoother (pipe wall) condition; furthermore, More's model goes out of scale and more deviations with respect of the experimental data set are observed with Colebrook's equation as well as with the models in [17] and [18]. This indicates the strong influence that pipe roughness has on the estimation of the friction factor.

Solutions provided in $[15,20]$ include a form of the $W$ function that might be the main source of deviation from the experimental data. On the contrary, the formulae for $f$ in [17] and that in [18] provide reasonable accuracy in their predictions for such factor. From these observations, it can be stated that when used properly, W function provides a useful tool to solve Colebrook's equation analytically rather than numerically. Figure 4, shows that Brkić's solutions $[18,19]$ are more accurate with respect to $(5)$ than Mikata's.

Furthermore, the use of analytical solutions of (5) not only proves the correctness of the equation, but it also provides reliable solutions to $f$ for an ample range of $\mathrm{Re}$ and pipe wall roughness conditions. Figure 5 , shows the departure from the experimental data set and the models obtained through the use of the $\mathrm{W}$ function.

The use of Lambert's W function offers a comprehensive tool to solve Colebrook's equation; however, to use it appropriately, the user needs to be familiar with how the $W$ function works and second, it requires understanding the fluid flow problem.

\subsection{Estimation of the friction factor using neural networks and artificial intelligence}

Another approach taken recently to calculate the friction factor in pipe networks is that of artificial intelligence. The main issue with this strategy to calculate the friction factor relies on how to "train" a neural network to give good estimates of $f$.

Parveen et al. [21] proposed the use of artificial intelligence (Al) to estimate the friction factor. They proposed this approach to avoid the use of recursive calculations needed to solve Colebrook's equation. The authors [21] tried different Al strategies to calculate the friction factor;
Fig. 3 Estimation of the friction factor using Lambert's W function and its comparison with Nikuradse [2] results and Colebrook [7] equation
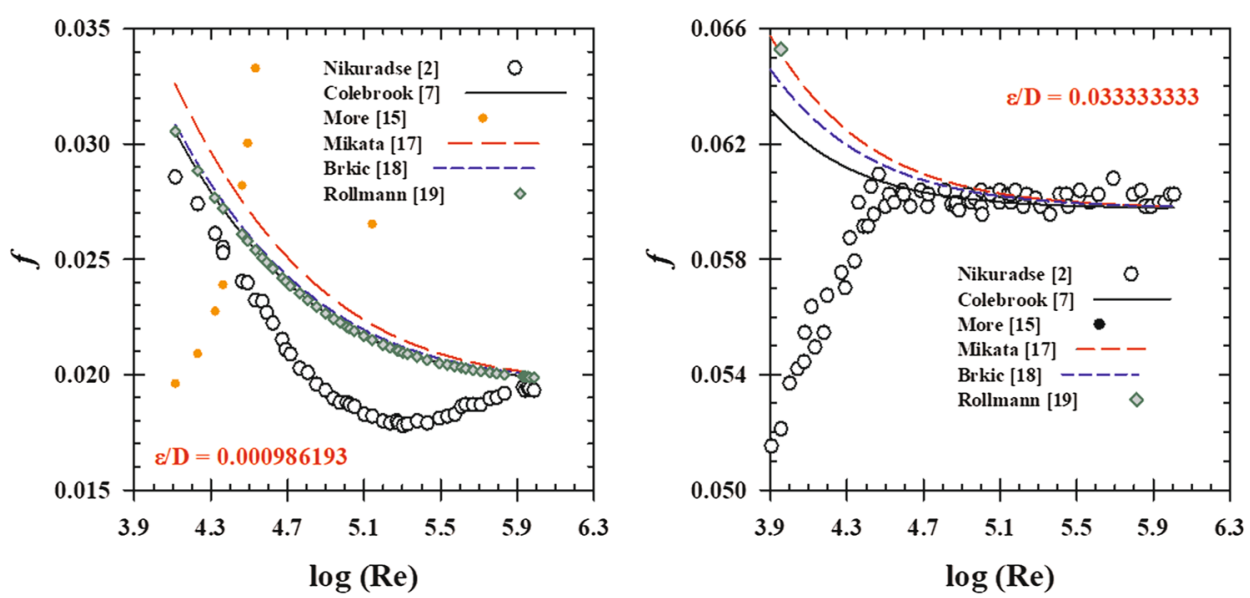
Fig. 4 Comparison between Mikata [17] and Brkić $[18,19]$ analytical solution with respect of Coelbrook [7] equation
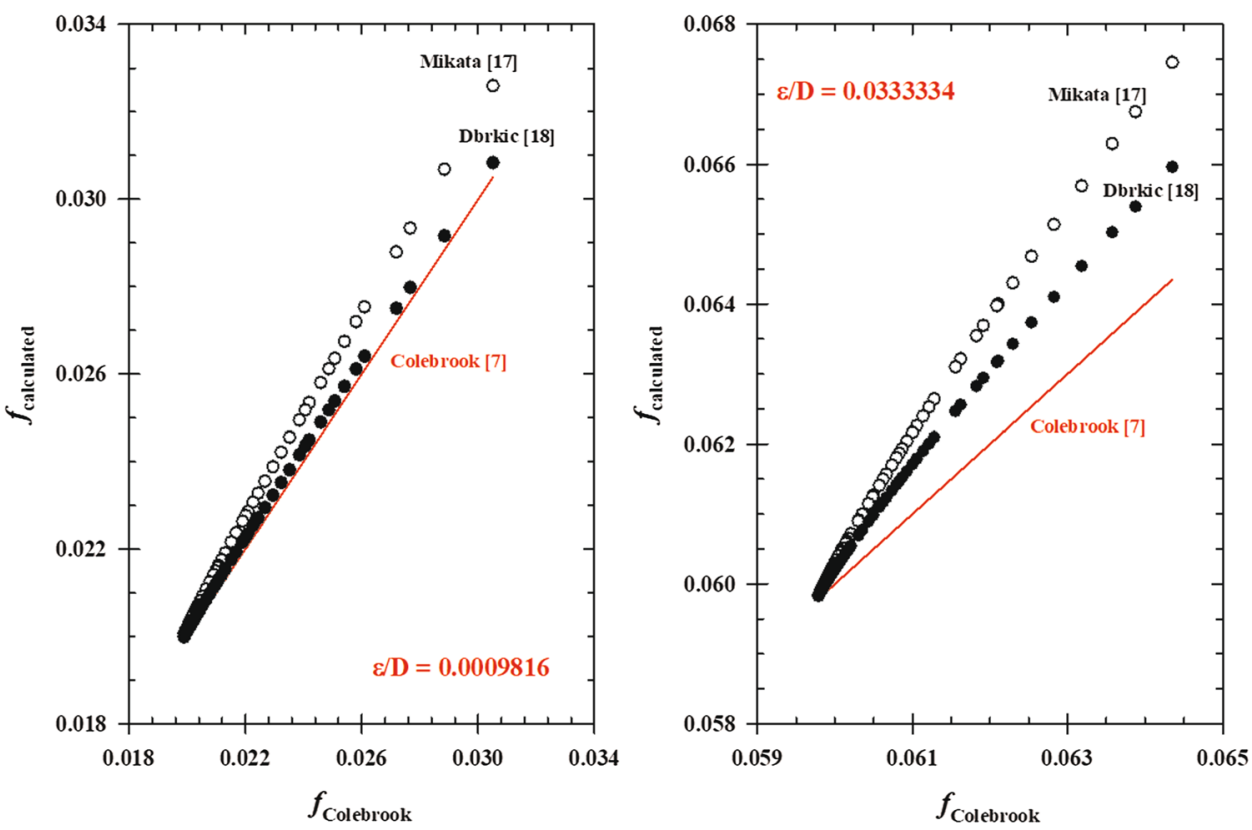

Fig. 5 Comparison between calculated values of $f$ using Lambert's W function and Nikuradse [2] data set
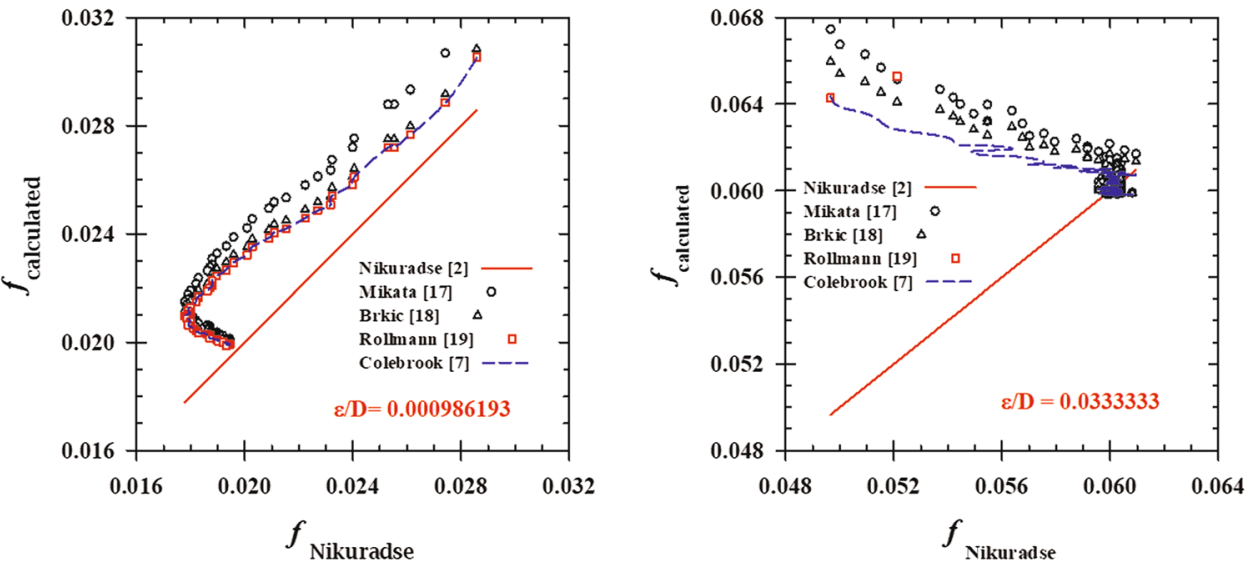

they found out that vector regression gives the best results.

Although this approach seems to work well, still the implementation of the neural network and its training to obtain the best values of $f$ is complex and if this is not done properly, this whole approach will not work.

Özger and Yildirim [22] proposed the use of adaptive neuro-fuzzy computational techniques to estimate the friction factor in pipe networks; their results are comparable with those obtained using (5) for distinct Re and $\varepsilon / D$ conditions. This Al approach works upon using logical statements; the technique does not require mathematical formulations and relies on establishing logical relationships between input and output variables.

Salmasi et al. [23] also calculated the friction factor by using extended neural networks. In this case, the authors used the gene expression programming approach. They noticed the extent of errors induced by this method, nevertheless, the results obtained seem to be satisfactory. Figure 6 shows the data obtained by this research and compares it with Nikuradse [2] and Swanson et al. [24] experimental data.

Results in Fig. 6, show that there is good correspondence among the calculated values of $f$ in [23] and the experimental data for smooth pipe, i.e. $\varepsilon / D \delta 0$ [24] and Nikuradse's [2] smoothest testing condition $(\varepsilon / D=0.00098619)$. On the other hand, for the rougher condition, the computed data set [23] shows the same behaviour with $\varepsilon / D=0.08$, like that of the experimentally, measured [2] with $\varepsilon / D=0.03333$; unfortunately, the data available from [23] does not match the experimental conditions in terms of the roughness reported in [2]. In spite of this, the Al computed values of the friction factor seems to work accordingly to the experimental results. 


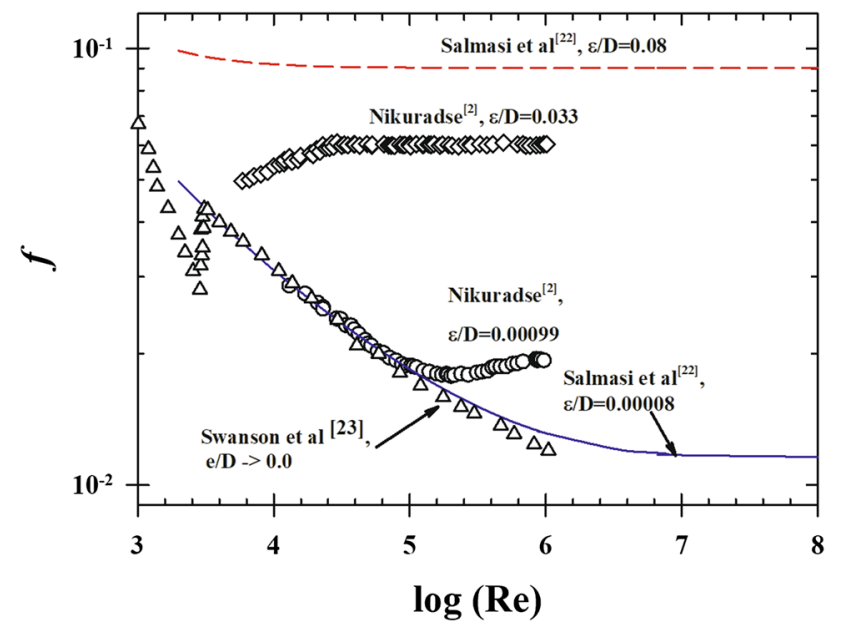

Fig. 6 Comparison of the friction factor calculated with artificial intelligence algorithms (Salmasi et al. [23]) and measured values of $f$ in a smooth pipe (Swanson et al. [24]) and pipes with different roughness (Nikuradse [2])

Samadianfard [25], used another Al technique to estimate the friction factor. In this work, gene expression programming was used. From this work, an explicit expression to determine the friction factor was obtained:
Table 3 Functions in Eq. (13) to calculate $f$

\begin{tabular}{ll}
\hline Function & Expression \\
\hline $\mathrm{a}$ & $\frac{64}{\mathrm{Re}}$ \\
$\mathrm{y}_{1}$ & $1-\frac{1048}{\frac{4.489}{10^{20}} \mathrm{Re}^{6}\left(0.148 \mathrm{Re}-\frac{2.306 \mathrm{Re}}{0.003133 \mathrm{Re}+9.646}\right)+1050}$ \\
$\mathrm{y}_{2}$ & $1.012-\frac{1}{0.02521(\mathrm{Re})\left(\frac{\varepsilon}{D}\right)+2.202}$ \\
$\mathrm{y}_{3}$ & $1-\frac{1}{0.000389\left(\mathrm{Re}^{2}\right)\left(\frac{\varepsilon}{D}\right)^{2}+0.0000239 \mathrm{Re}+1.61}$ \\
$\mathrm{c}_{1}$ & $\frac{0.316}{\mathrm{Re}^{0.25}}$ \\
$\mathrm{c}_{3}$ & $\frac{0.25}{\log \left(\frac{1}{3.71} \frac{\varepsilon}{D}\right)^{2}}$ \\
\hline
\end{tabular}

On the other hand, the c's functions in (13) link the different $y$ functions. These functions are of different natures of the $y$ 's ones. They depict the hydrodynamic regime and are estimated from either Blasius or von Kárman logarithmic velocity distributions, such as (3). Lastly, a function relates to the laminar flow regime and is equivalent to Eq. (1). Table 3 shows the functions embedded in (13). Equations (12) and (13) are compared to the Nikuradse data set in Fig. 7.

$f=\frac{\operatorname{Re}^{\frac{\varepsilon}{D}}-0.6315093}{\operatorname{Re}^{\frac{1}{3}}+\operatorname{Re} \frac{\varepsilon}{D}}+0.0275308\left(\frac{6.929841}{\operatorname{Re}}+\frac{\varepsilon}{D}\right)^{\frac{1}{9}}+\left(\frac{10^{\frac{\varepsilon}{D}}}{\frac{\varepsilon}{D}+4.781616}\right)\left(\sqrt{\frac{\varepsilon}{D}}+\frac{9.99701}{\operatorname{Re}}\right)$

By extending his work, Samadianfard et al. [26], improved the accuracy of (12), resulting in an expression far more complex than (12). To get to such a level of exactness, the neural network needed to estimate the friction factor besides involving more complicated mathematical operations, it also grew substantially.

In another report [27], it is proposed to calculate the friction through the use of a unified approach to obtain a single expression to estimate $f$, such expression is:

$f=a\left(1-y_{1}\right)+c_{1}\left(y_{1}-y_{3}\right)+c_{3} y_{2}$

Equation (13) links the different hydrodynamic regimes obtaining a continuous representation of $f$ for several values of $\operatorname{Re}$ and $\varepsilon / D$. For this solution scheme to work, the authors used a combination of independent functions. The terms in (13) all of them are independent functions that represent different flow characteristics. The $y$ 's functions in (13) are called switching functions; they smoothly transition the different hydrodynamic flow regimes. They are generated with the use of HeuristicLab software [28], which uses heuristic and evolutionary algorithms for different Al applications. The $y$ 's functions are sigmoid in nature and are presented in Table 3.
From the plots in Fig. 7, it is clear that (13) offers a better representation of the friction factor than (12); this is particularly more evident for high roughness. This figure along with the data shown in Fig. 6 demonstrates the potential use of Al programming techniques to evaluate the friction factor, particularly in big pipe networks. The computation of this factor can be speeded up significantly with the use of Al algorithms; however, the chief concern with this approach definitely relies on the experience of the programmer.

If the person who is to set up artificial intelligence (the neural network, symbolic regression) to compute $f$ is not familiar with the hydrodynamics of the system to calculate, then serious errors could be induced and wrong values will be obtained. For this reason, errors of new models should be verified by numerical experiments.

Additionally, if specific expressions to determine $f$ are produced through $\mathrm{Al}$ algorithms, these expressions [such as (12) and (13)] are far more complex than those developed from the integration of (3) and those obtained through the use of $W$ function. 
Fig. 7 Comparison of calculated values of $f$ with expressions obtained through artificial intelligence algorithms (Samadianfard [25], Brkić [2]) and measured values (Nikuradse [2])
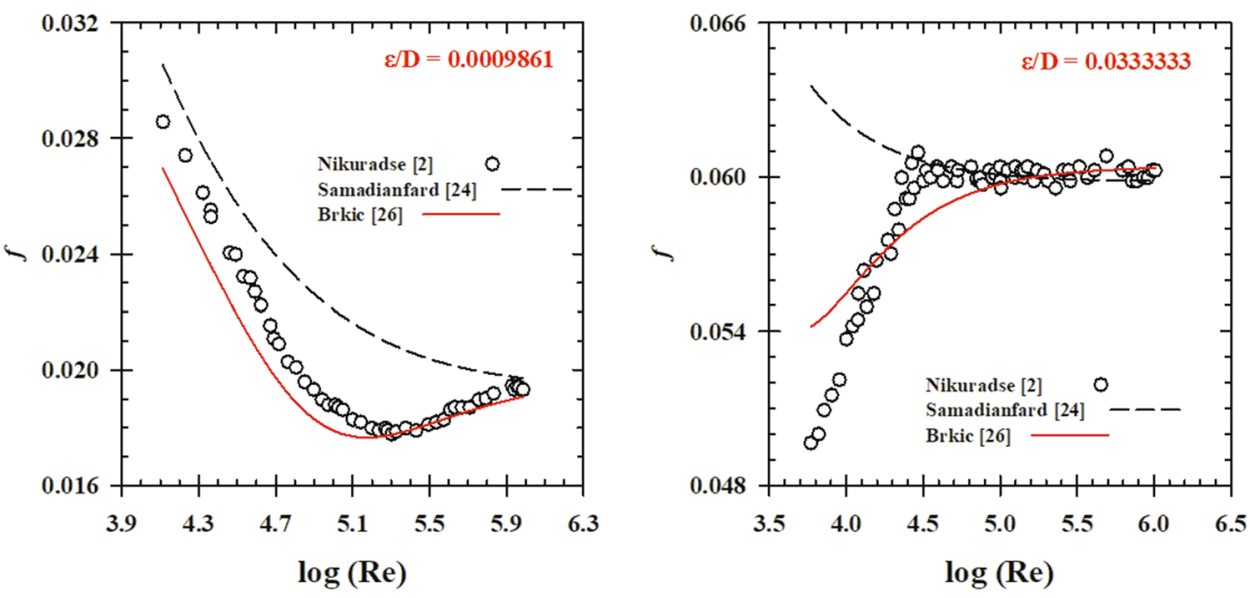

\section{Phenomenological approach}

In view of the different alternatives to compute the friction factor, these authors presented [9] not long ago a new analytical model to calculate the friction factor under a wide range of both $\mathrm{Re}$ and $\varepsilon / \mathrm{D}$ conditions.

These authors believe that this model is better suited to calculate $f$ since it was conceived upon modelling the laminar to turbulent transition. This fact is quite important; Colebrook model and the others obtained through integration of the logarithmic velocity distribution fail to include (directly) the effect of turbulence on the friction factor.

It can be seen in Moody's chart that within the laminar flow regime, the friction factor decreases as Re increases. Upon getting to the laminar to turbulent transition, the friction factor value tends to increase (irrespective of the pipe roughness) to a maximum value and as Re comes close to $4 \times 10^{3}$, the friction factor drops progressively until reaching a stable value, which is no longer affected by Re. Furthermore, the wall roughness seems to have a stronger effect on $f$ rather than Re as the flow becomes fully turbulent.

In this regard, the Díaz-Plascencia model [9] succeeds where previous models have failed. The key feature of this model is that it assumes the laminar to turbulent flow transition as a sigmoid type of function that results from the randomness of the flow associated with the turbulence. This element of the model allows for pursuing the best accuracy in the different models and strategies (W function, $\mathrm{Al}$ ) used to compute the friction factor.

Upon further work on this model, the authors have modified (an upcoming publication offers specific details) the original equation reported in [9] to the following form:

$$
f=\frac{64}{\operatorname{Re}}+\frac{\lambda_{1}}{1+\exp \left(\frac{\tau_{1}-\operatorname{Re}}{100}\right)}+\frac{\lambda_{2}}{1+\exp \left(\frac{\tau_{2}-\operatorname{Re}}{150} \times \frac{\varepsilon}{D}\right)}
$$

Equation (14) is similar to (13) in the sense that it is comprised of independent functions working together. The first term of (14) represents the friction factor under the laminar flow regime; the second term includes $\lambda_{1}$ and $\tau_{1}$ that are parameters of a sigmoid function that characterizes the laminar to turbulent regime transition; these two parameters are constant and have values of $\lambda_{1}=0.02$ and $\tau_{1}=3000$. This transition relates to the boundary layer present next to the pipe wall; as this transition takes place, the sudden increase in the friction factor occur; as the flow accelerates, the boundary layer attached to the pipe wall thins and the influence of the roughness of the pipe becomes more important, as the flow becomes fully turbulent, the friction factor tends to stabilize. This last feature is also accounted for in the last term of (14), which also is of sigmoid nature but it implies the effect of the roughness on the friction factor. The parameters $\lambda_{2}$ and $\tau_{2}$ accounts for the Reynolds and roughness effects respectively. Table 4 shows the parameters and functions used in (14).

Table 4 Parameters and functions in Eq. (14) to compute $f$

\begin{tabular}{ll}
\hline Parameter/function & Value/expression \\
\hline$\lambda_{1}$ & 0.02 \\
$\tau_{1}$ & 3000 \\
$\lambda_{2}$ & $\left|\lambda_{1}-\left(\frac{1}{-2 \log \left(\frac{1}{3.71} \frac{\varepsilon}{D}\right)}\right)^{2}\right|$ \\
$\tau_{2}$ & $\tau_{2}=\frac{0.77505}{\left(\frac{\varepsilon}{D}\right)^{2}}-\frac{10.984}{\frac{\varepsilon}{D}}+7953.8$
\end{tabular}


Considerations made to obtain (14) are similar to those in [27]. The main difference between (13) and (14) relies on the manner in which the respective functions in these expressions were constructed. Whereas Brkić and Praks [27] used heuristic algorithms (based on Al), these authors focused on modelling the change of flow regime phenomenon using independent experimental data sets $[2,24,29]$ and theoretical models well established.

This flow modelling approach seems to be the best manner to describe not only the friction factor but more importantly the flow transition from laminar to the turbulent regime, In addition to that, it seems that this modelling approach is gaining adepts since other works in this regard have been published lately $[30,31]$.

Avci and Karagoz [30] set out to model the friction factor by balancing out the kinetic energy that a flow experiences as it gains momentum, transitioning from the laminar to the turbulent flow.

Similar to these authors, they proposed an expression for that factor comprising the laminar and turbulent flows, their proposed equation contains the laminar flow friction factor $\left(f_{l}=64 / \mathrm{Re}\right)$ term, whereas, the turbulent flow contribution $\left(f_{t}\right)$ to the friction factor is a complex function. The effect of wall roughness is added through an empirical constant $(\mathrm{Cm})$, their suggested equation is:
$f=f_{t}+\left(\frac{64}{\operatorname{Re}}-f_{t}\right) \exp \left(-\left(\frac{C_{m} \operatorname{Re}}{2560}\right)^{8}\right)$

On the other hand, Kaltenbacher et al. [31] modelled the laminar to turbulent transition particularly. They did so by assuming the flow as two dimensional and depending upon the pressure drop and the pipe wall roughness. They proposed the use of a smooth $(\gamma)$ function that allows for the flow transition. This $\gamma$ function, in turn, is comprised of different "smaller" functions working to stabilize the flow transition.

These novel results [30,31] are in good agreement with experimental sets $[2,24]$. Furthermore, these works offer similar results to those by these authors [9], and Brkić and Praks [27].

Figure 8 compares the results for $f$ from the Díaz-Plascencia model with some of the solutions obtained for this factor using some of the solution schemes depicted in this work. It can be seen in this figure that Eqs. (13) and (14) offer the best approximations to Nikuradse's experimental results. The other models tested show almost the same deviations from the experimental data. Table 5 shows the relative error for the different models compared in Fig. 8. The least relative errors are obtained by Eqs. (13) and (14) respectively.
Fig. 8 Comparison of the computed friction factor using distinct calculation schemes with respect of Nikuradse [2] data
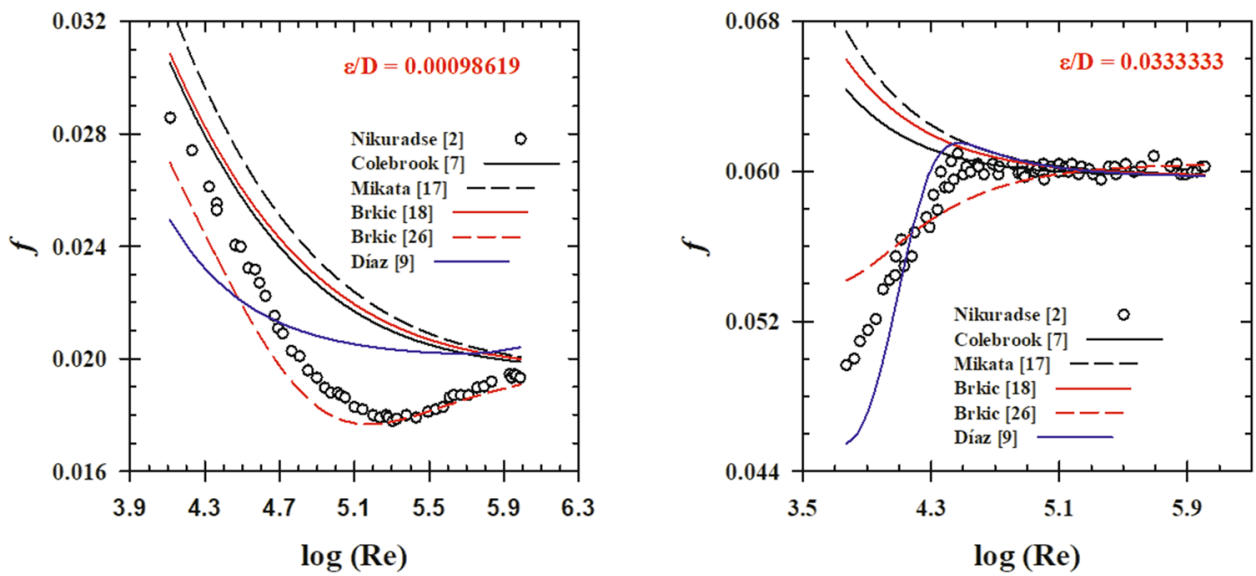

Table 5 Relative error with respect of Nikuradse [2] results for different models tested

\begin{tabular}{llll}
\hline Model & Solution strategy & $\begin{array}{l}\text { \% error } \\
(\varepsilon / D=0.00098619)\end{array}$ & $\begin{array}{l}\% \text { error } \\
(\varepsilon / D=0.033333)\end{array}$ \\
\hline Colebrook [7] & $\begin{array}{l}\text { Integration of logarithmic veloc- } \\
\text { ity distribution }\end{array}$ & 11.57 & 4.02 \\
Mikata [17] & Lambert's W function & 15.24 & 5.25 \\
Brkić [18] & Lambert's W function & 12.72 & 4.72 \\
Brkić [26] & Artificial intelligence & 3.72 & 1.64 \\
Díaz-Plascencia [9] & Phenomenological approach & 8.58 & 1.96 \\
\hline
\end{tabular}


To quantify the deviation from the measured $f$ values, the relative error was computed using:

$\%$ error $=100 \times\left|\frac{f_{\text {computed }}-f_{\text {Nikuradse }}}{f_{\text {Nikuradse }}}\right|$

The strategies shown to compute the friction factor show distinct degrees of accuracy with respect to Nikuradse's data sets.

To further test some of the different tools used to compute the friction factor, in Fig. 9 we compare the friction factor calculated with Colebrook's equation (Eq. 5) with the models shown in Eqs. (9), (10a), (12), (13) and (14). These models have been tested over larger $\operatorname{Re}$ and $\varepsilon / D$ ranges of values. The Reynolds number varied from $10^{3}$ to $10^{8}$; whereas the pipe wall roughness varied from 0.0009 (very smooth) to 0.08 (rougher).

In the plots shown in Fig. 9, it can be seen that the models represented by Eqs. (5), (9), (10a) and (12) show the same trend. As the Reynolds number increases from $10^{3}$ to roughly $10^{4}$, the friction factor calculated with these expressions decreases continuously until stabilizing at $\operatorname{Re}>10^{4}$. This behaviour is observed in every $\varepsilon / D$ value used to carry out the calculations. No indication of transitioning from the laminar to the turbulent flow regime is indicated by this set of models. This fact seems to limit the predictive capacity of these models.

On the contrary, the Brkić and Praks [27] and Díaz and Plascencia [9] models, they clearly indicate the transition from laminar to turbulent flow that is expected as the Re increases from $10^{3}$ to $10^{4}$, regardless of the roughness of the pipe wall. Furthermore, such transitions seem to occur at around $\operatorname{Re} \sim 3.5 \times 10^{3}$, and it is consistent throughout the whole tested interval.

The laminar to turbulent transition is more abrupt as the pipe wall roughness values increases. Once the turbulent regime takes place and stabilizes, it is clear that the values for the friction factor calculated with Eqs. (13) and (14) are in very good agreement with the other friction factor values calculated with the other models cited.

Table 6, compares the error \% that the different models evaluated in this work have with respect of Colebrook's equation. The error \% was calculated using Eq. (15), using $f$ computed with Eq. (5) as reference.

The data in Table 6 shows the models presented in references $[17,19,25]$ are in very good agreement with Colebrook's model. On the other hand, Brkić and Praks unified formula and the model proposed by these authors present the higher error \% respect to Colebrook's compared to the other models tested. The Brkić and Praks unified formula
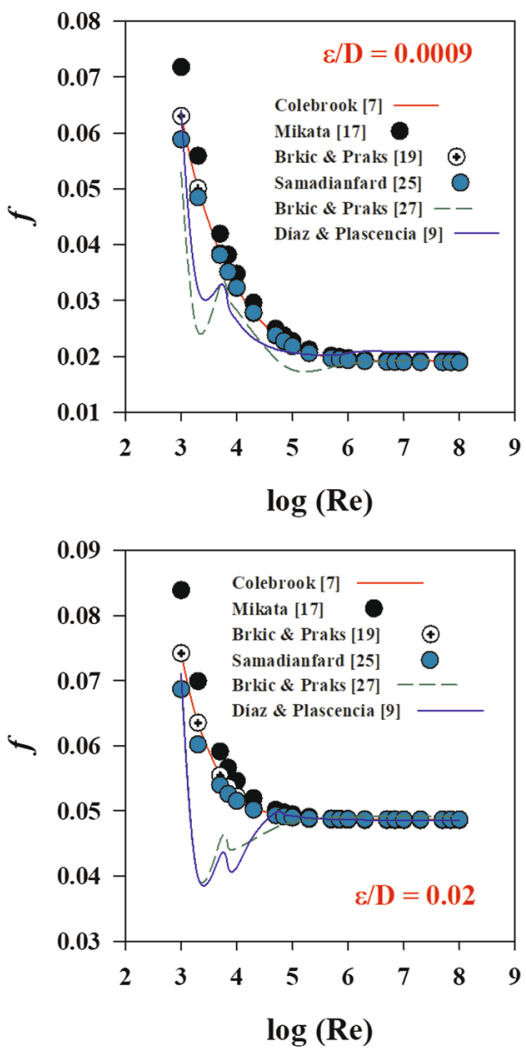
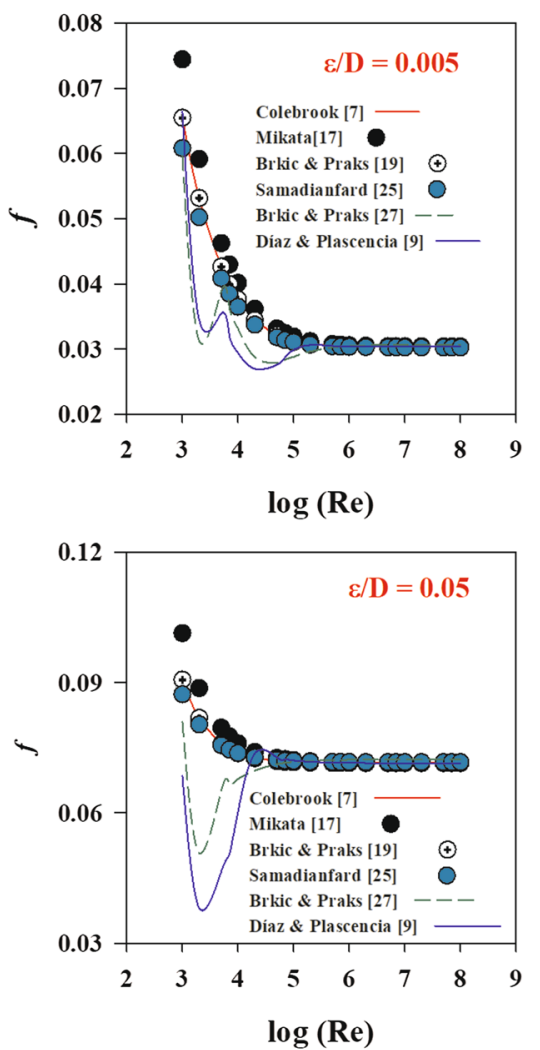
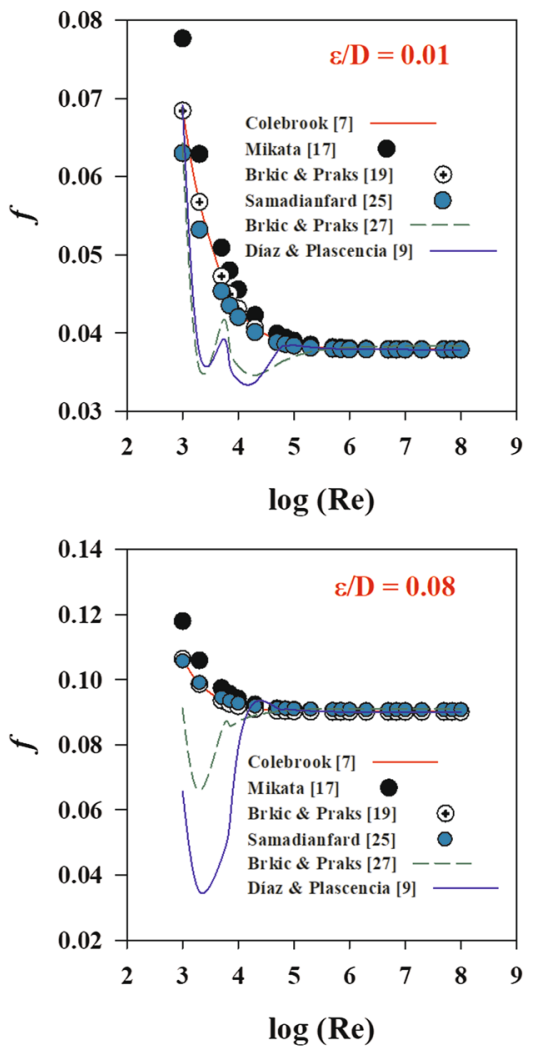

Fig. 9 Comparison of different models to calculate the friction factor with respect to the Colebrook [7] equation 
Table 6 Relative error with respect of Colebrook's [7] results for different models tested

\begin{tabular}{lclllll}
\hline Reference & $\begin{array}{l}\text { \% error } \\
\varepsilon / D=0.0009\end{array}$ & $\begin{array}{l}\text { \% error } \\
\varepsilon / D=0.005\end{array}$ & $\begin{array}{l}\text { \% error } \\
\varepsilon / D=0.01\end{array}$ & $\begin{array}{l}\text { \% error } \\
\varepsilon / D=0.02\end{array}$ & $\begin{array}{l}\text { \% error } \\
\varepsilon / D=0.05\end{array}$ & $\begin{array}{l}\text { \% error } \\
\varepsilon / D=0.08\end{array}$ \\
\hline Mikata [17] & 3.73 & 2.96 & 2.61 & 2.22 & 1.71 & 1.47 \\
Brkić and Praks [19] & 0.08 & 0.10 & 0.11 & 0.12 & 0.15 & 0.17 \\
Samadianfard [25] & 1.04 & 1.65 & 1.37 & 0.92 & 0.18 & 0.63 \\
Brkić and Praks [27] & 10.02 & 6.01 & 5.42 & 4.80 & 3.61 & 2.88 \\
Díaz and Plascencia [9] & 2.13 & 6.90 & 5.49 & 5.51 & 7.86 & 9.83 \\
\hline
\end{tabular}

[27] provides a new unified expression for Newtonian fluids valid for all pipe flow regimes from laminar to fully rough turbulent flow. This equation follows the inflectional form of curves suggested in Nikuradse's experiment rather than the monotonic shape proposed by Colebrook and White. For this reason, it is obvious that the this new unified formula has a larger error than the simple model of Colebrook. Similarly, the Díaz and Plascencia model, shows and represents with accuracy the inflections shown by Nikuradse's data.

The novel formulas should be tested with respect to the Nikuradse's data set.This is due to the fact that Eqs. (13) and (14), take into account the effect of the flow transition on the pipe wall roughness; whereas the models in Eqs. (5), (9), (10a) and (12) do not do so. Because of this these models behave in the same manner. Moreover, the good fit between Mikata's model and Colebrook's is due to Eq. (9) is proposed as an analytical solution to Eq. (5). Equations (10a) and (12) were formulated using Al strategies that not necessarily take into account the physics behind the flow problem. Because of this feature, these models show a higher departure from Nikuradse's data set, as indicated in Table 5.

In this regard, it is clear that the best approach to model the friction factor is that of considering the laminar-turbulent transition in the model formulation or what we call the phenomenological approach. This is a considerable contribution to the field of flow friction.

\section{Final remarks}

The calculation of the friction factor can be done in many different manners. It has been shown that the different strategies used to calculate the factor offer relatively similar results.

Besides the classic Colebrook model, other alternatives such as the use of Lambert W function and artificial intelligence training/programming are available to determine the friction factor. These other approaches offer similar results than classic models; however, their implementation requires experience in both the fluid dynamics problem and the actual computing procedure. If the person who decides to use these approaches is inexpert in both, it will induce considerable errors in the calculation of this factor.

Modelling the flow behaviour as it transitions from laminar to turbulent seems to be the best approach to calculate the friction factor under different Re and pipe wall roughness conditions. This not only gives the best estimations for the friction factor but allows for a better understanding of the physics behind the change from laminar to turbulent flow regime.

\section{Compliance with ethical standards}

Conflict of interest There is no conflict of interest to report.

\section{References}

1. de Nevers $\mathrm{N}$ (2005) Fluid mechanics for chemical engineers, 3rd edn. McGraw-Hill, NewYork

2. Nikuradse J (1950) Laws of flow in rough pipes. NACA TM 1292, Washington

3. von Kárman T (1931) Mechanical similitude and turbulence. NACA TM 611, Washington

4. Blasius H (1913) Das aehnlichkeitsgesetz bei reibungsvorängen in flüssigkeiten. Springer, Berlin

5. von Mises R (1914) Elemente der technischen hydrodynamik. Teuber, Leipzig

6. Colebrook CF, White CM (1937) Experiments with fluid friction in roughened pipes. Proc $R$ Soc A 161:367-381

7. Colebrook CF (1939) Turbulent flow in pipes with particular reference to the transition region between the smooth and rough pipe laws. J Inst Civ Eng 11:133-156

8. Moody LF (1944) Friction factors for pipe flow. Trans ASME 66:671-684

9. Díaz-Damacillo L, Plascencia G (2019) A new six parameter model to estimate the friction factor. AIChE J 65(4):1144-1148

10. Romeo E, Royo C, Monzón A (2002) Improved explicit equations for estimation of the friction factor in rough and smooth pipes. Chem Eng J 86:369-374

11. Haaland SE (1983) Simple and explicit formulas for the friction factor in turbulent pipe flow. J Fluids Eng 105:89-90

12. Zigrang DJ, Sylvester ND (1982) Explicit approximations to the solution of Colebrook's friction factor equation. AIChE J 28(3):514-515

13. Churchill SW (1977) Friction-factor equation spans all fluid-flow regimes. Chem Eng 84:91-92 
14. Shaikh MM, Massan SR, Wagan AI (2015) A new explicit approximation to Colebrook's friction factor in rough pipes under highly turbulent cases. Int J Heat Mass Transf 88:538-543

15. More AA (2006) Analytical solutions for the Colebrook and White equation and for pressure drop in ideal gas flow in pipes. Chem Eng Sci 61:5515-5519

16. Corless RM, Gonnet GH, Hane DEG, Jeffrey DJ, Knuth DE (1996) On the Lambert W function. Adv Comput Math 5:329-359

17. Mikata Y, Walczak WS (2016) Exact analytical solutions of the Colebrook-White equation. J Hydraul Eng 142(2):040150501-040150506

18. Brkić D (2011) Review of explicit approximations to the Colebrook relation for flow friction. J Pet Sci Eng 77:34-48

19. Brkić D, Praks P (2019) Accurate and efficient explicit approximations of the Colebrook flow friction equation based on the Wright $\omega$-function. Mathematics 7(34):1-15

20. Rollmann P, Spindler K (2015) Explicit representation of the implicit Colebrook-White equation. Case Stud Therm Eng 5:41-47

21. Parveen N, Zaidi S, Danish M (2019) Artificial intelligence (AI)based friction factor models for large piping networks. Chem Eng Commun 206:1-18

22. Özger M, Yildirim G (2009) Determining turbulent flow friction coefficient using adaptive neuro-fuzzy computing technique. Adv Eng Softw 40:281-287

23. Salmasi F, Kathibi R, Ghorbani MA (2012) A study of friction factor formulation in pipes using artificial intelligence techniques and explicit equations. Turk J Eng Environ Sci 36:121-138
24. Swanson CJ, Julian B, Ihas GG, Donnelly RJ (2002) Pipe flow measurements over a wide range of Reynolds numbers using liquid helium and various gases. J Fluid Mech 461:51-60

25. Samadianfard $S$ (2012) Gene expression programming analysis of implicit Colebrook-White equation in turbulent flow friction factor calculation. J Pet Sci Eng 92-93:48-55

26. Samadianfard S, Sattari MT, Kisi O, Kasemi H (2014) Determining flow friction factor in irrigation pipes using data mining and artificial intelligence approaches. Appl Artif Intell 28:793-813

27. Brkić $D$, Praks $P$ (2018) Unified friction formulation from laminar to fully rough turbulent flow. Appl Sci 8(2036):1-13

28. https://dev.heuristiclab.com/trac.fcgi/. Accessed 10 Dec 2019

29. McKeon BJ, Swanson CJ, Zagarola MV, Donnelly RJ, Smits AJ (2004) Friction factors for smooth pipe flow. J Fluid Mech 511:41-44

30. Avci A, Karagoz I (2019) A new explicit factor formula for laminar, transition and turbulent flows in smooth and rough pipes. Eur J Mech B Fluids 78:182-187

31. Kaltenbacher S, Steinberger M, Horn M (2019) Transitional water flow in steady-state. Appl Math Model 77:478-490

Publisher's Note Springer Nature remains neutral with regard to jurisdictional claims in published maps and institutional affiliations. 